\title{
A statistical approach to spectrum sensing using bayes factor and p-values
}

\author{
Deepa N. Reddy ${ }^{1}$, Y. Ravinder ${ }^{2}$ \\ ${ }^{1}$ Department of Electronics and Telecommunication Engineering, JSPM's Rajarshri Shahu College of Engineering, \\ Savitribai Phule Pune University, India \\ ${ }^{1}$ Department of Electronics and Communication Engineering, B.M.S Institute of Technology and Management, India \\ ${ }^{2}$ Department of Electronics and Telecommunication Engineering, Pune Institute of Computer Technology, Savitribai \\ Phule Pune University, India
}

\begin{tabular}{l} 
Article Info \\
\hline Article history: \\
Received Sep 10, 2018 \\
Revised Apr 4, 2019 \\
Accepted Apr 9, 2019 \\
\hline Keywords: \\
Bayes factors \\
Diversity combiners \\
Meta-analytic approach \\
Probability of detection \\
p-value \\
Spectrum sensing
\end{tabular}

\begin{abstract}
The sensing methods with multiple receive antennas in the Cognitive Radio (CR) device, provide a promising solution for reducing the error rates in the detection of the Primary User (PU) signal. The received Signal to Noise Ratio at the CR receiver is enhanced using the diversity combiners. This paper proposes a statistical approach based on minimum Bayes factors and pValues as diversity combiners in the spectrum sensing scenario. The effect of these statistical measures in sensing the spectrum in a CR environment is investigated. Through extensive Monte Carlo simulations it is shown that this novel statistical approach based on Bayes factors provides a promising solution to combine the test statistics from multiple receiver antennas and can be used as an alternative to the conventional hypothesis testing methods for spectrum sensing. The Bayesian results provide more accurate results when measuring the strength of the evidence against the hypothesis.
\end{abstract}

Copyright $\odot 2019$ Institute of Advanced Engineering and Science. All rights reserved.

\section{Corresponding Author:}

Deepa N. Reddy,

Department of Electronics and Telecommunication Engineering,

JSPM's Rajarshri Shahu College of Engineering,

Savitribai Phule Pune University,

Pune-411046, India.

Email: reddydeepa2680@gmail.com

\section{INTRODUCTION}

Spectrum sensing in Cognitive Radio (CR) is a binary hypothesis testing problem. It is the decision on the Signal absent, noise-only (null) hypothesis denoted by $\mathrm{H}_{0}$ and the signal-present (alternate) hypothesis denoted by $\mathrm{H}_{1}$. Energy Detection (ED) is a very simple technique that decides on hypothesis $\mathrm{H}_{0}$ or $\mathrm{H}_{1}$ using the average energy in the observations as the decision statistic. The threshold chosen for ED is dependent on the noise power. This makes the performance of the ED sensitive to uncertainty in the noise variance, especially at low Signal to Noise Ratio (SNR) [1]. Diversity techniques are employed to mitigate the effect of small scale fading and hence provide improvement in the received SNR to achieve higher probability of detection $[2,3]$.

From the studies, the maximal ratio combining (MRC), equal gain combining (EGC), and selection combining (SC) are the most commonly used diversity combiners. These diversity combining techniques no doubt provide improvement in the receive SNR but they demand the learning of the channel state information (CSI). Hence this increases the implementation complexity [3-7].

Several diversity combining techniques were proposed in the literature which would mitigate the impact of the channel estimation error on the performance of diversity receivers. The non-coherent combining schemes which do not need the CSI are investigated in $[1,7]$. Under this category the square law 
combiner (SLC) and square law selection (SLS) are studied which produces the decision statistic using the outputs of the square-law devices available in each of the diversity branch. In [8] the use of evolutionary algorithms on receiver diversity based on the imperialistic competitive algorithm (ICA) is proposed. It is shown that this combiner does not demand the CSI, and it provides superior performance compared to MRC.

The Goodness of fit test based sensing (GoFT) using Anderson Darling (AD) and Jarque Bera (JB) tests check for the distribution of test statistic under null hypothesis and is independent of the noise distribution [9-11]. These statistical methods were primarily meant to measure the strength of evidence for drawing an accurate decision in hypothesis testing based on a sample. The use of these statistical methods in spectrum sensing is investigated in previous studies but the studies do not focus on receiver diversity using multiple antennas at the CR device.

In this paper two benchmarks coined as p-Value and Minimum Bayes factor (MBF) are used in decision making in hypothesis testing. The first metric, p-Value is viewed as an index of the "strength of evidence" against $\mathrm{H}_{0}$, with small $\mathrm{p}$ indicating an unlikely hypothesis [12] and is widely used in medical research and decision-making. Using the p-Value the compatibility of the data with the null hypothesis is measured. The second metric is the Bayes factor and is often referred to as the "strength of evidence" or "weight of evidence". The Minimum Bayes factor provides the smallest amount of evidence that can be stated for the null hypothesis. The sound theoretical foundation of the MBF and its interpretation allows its usage in both inference and decision making. They have straightforward interpretation as the strength of the evidence in favor of $\mathrm{H}_{1}$ relative to $\mathrm{H}_{0}$ [13-16]. This work is the first of its kind in which the use of $\mathrm{p}$ values and Minimum Bayes factors are proposed in the context of spectrum sensing. The paper is organized as follows: Section 2 gives the overview of blind sensing schemes for primary user detection, Section 3 discusses the proposed method, Section 4 discusses the results and Section 5 concludes the paper.

\section{SYSTEM MODEL AND PROBLEM FORMULATION}

Consider the scenario of Single Input Multiple Output (SIMO) system with one transmit antenna and multiple receiver antennas. Assume that each CR contains $M$ antennas. The $M$ diversity branches are assumed to be sufficiently far from each other. Hence this study takes full advantage of this assumption that the received signals are statistically independent and the correlation among them is considered to be negligible. Corresponding to the signal received in the $i^{\text {th }}$ antenna of the CR device the hypotheses $H_{0}$ and $H_{l}$ are defined as

$$
\begin{aligned}
& H_{0}: x_{i}[k]=v_{i}[k] \\
& H_{I}: x_{i}[k]=h s[k]+v_{i}[k]
\end{aligned}
$$

where, $h$ is the amplitude gain of the channel, $i$ is the antenna index $(i=1,2, . . M)$ at each $\mathrm{CR}, s[k]$ is the transmitted signal by PU and $v_{i}[k]$ is the AWGN noise component.

\subsection{Existing blind sensing methods}

\subsubsection{Square law detector}

Energy detector (ED) or Square law detector is the most commonly used method for hypothesis testing in a CR environment. Each individual branch at the receiver is provided with an energy detector to provide the instantaneous individual branch energy measurements. The energy of the received signal at the $i^{\text {th }}$ branch is $Y_{i}$ and $N$ is the sample size. The decision static $Y_{i}$ is compared against a fixed threshold $\lambda$.

$$
Y_{i}=\sum_{k=1}^{N}\left|x_{i}[k]\right|^{2}
$$

The simple hypothesis testing problem is formulated in Equation (3) as

$$
Y_{i}^{\stackrel{H_{1}}{\underset{ }{<}}} \underset{H_{0}}{<} \lambda
$$

The operation in Equation (2) is executed using a square law device provided at each diversity branch of the $\mathrm{CR}$ receiver. The following conventional square law combining techniques are used to form a better estimate of the primary user signal [7]. 


\section{a. Square-law selection}

The energy vectors from $M$ diversity branches, $Y_{l}, Y_{2}, \cdots, Y_{M}$ are used in SLS. The branch with the highest energy is chosen. The test statistic is given as

$$
Y_{s l s}=\max \left(Y_{1}, Y_{2}, \ldots . Y_{M}\right)
$$

\section{b. Square law combining}

The energy vectors from $M$ diversity branches, $Y_{1}, Y_{2}, \cdots, Y_{M}$ are gathered and combined in SLC to make a combined decision. The test statistic is as

$$
Y_{\text {slc }}=\sum_{i=1}^{M} Y_{i}
$$

\subsubsection{Goodness of fit tests based sensing}

Another blind sensing method is the goodness of fit tests (GoFT). These tests are blind nonparametric hypothesis testing method, which decides on the null hypothesis if the received samples follow the noise Cumulative Distribution Function (CDF) denoted as $\mathrm{F}_{0}$. Let $x[k]$ denote the set of $\mathrm{N}$ discrete time vector observations $k=1,2 \ldots . N$. Let the $i^{t h}$ component of $x[k]$ be denoted as $x_{i}[k], i=1,2 \ldots M$. The signal detection in noise is therefore given as a simple hypothesis testing problem in [9-11] and is expressed as

$$
\begin{aligned}
& \text { Decide on } H_{0} \text { : if } F_{n}(x)=F_{0}(x) \\
& \text { Decide on } H_{1} \text { : if } F_{n}(x) \neq F_{0}(x)
\end{aligned}
$$

where, $F_{n}(x)$ is the empirical CDF of the received sample. The popular goodness of fit tests are:

\section{a. Anderson darling (AD) test} is given as:

To test the normality of a random sample $\mathrm{x}[\mathrm{k}]$ the Anderson Darling test statistic formulated in [17]

$$
\mathrm{A}_{\mathrm{n}}{ }^{2}=-N-\frac{\sum_{k=1}^{N}(2 \mathrm{k}-1)\left(\ln \mathrm{z}_{\mathrm{k}}-\ln \mathrm{z}_{(\mathrm{N}+1-\mathrm{k})}\right)}{N}
$$

when the mean and variance of the sample are unknown the adjusted AD statistic as given in [18] is

$$
\mathrm{A}=\mathrm{A}_{\mathrm{n}}^{2}\left(1+\frac{0.75}{N}+\frac{2.25}{N^{2}}\right)
$$

where $\mathrm{z}_{\mathrm{k}}=\mathrm{F}_{0}\left(\mathrm{y}_{\mathrm{k}}\right)$ is the assumed distribution, $\mathrm{N}$ denotes the sample size, $\mathrm{ln}$ is the natural logarithm with $y_{k}=\left(x_{i}-\check{x}\right) / S \quad$ where $\check{x}=\sum^{x_{k}} /{ }_{N}$ and $S^{2}=\sum\left(x_{k}-\check{x}\right)^{2} /(N-1)$. The spectrum sensing problem is expressed as:

$$
\begin{aligned}
& H_{0}: A \leq \lambda_{c v} \\
& H_{l}: A>\lambda_{\mathrm{cv}}
\end{aligned}
$$

where, $\lambda_{c v}$ is a critical value. If $A$ exceeds the critical value then $H_{0}$ is rejected. A table of thresholds for different values of $P_{f}$ is given in [19].

\section{b. Kolmogorov-smirnov (KS) test}

In the KS test the distance between Fn (x) and F0 (x) is given by:

$$
\mathrm{D}_{\mathrm{n}}=\max \left|F_{n}(x)-F_{0}(x)\right|
$$

where $F_{n}(x)$ is the empirical distribution. If the samples under test are coming from $\mathrm{F} 0(\mathrm{x})$, then, $\mathrm{D}_{\mathrm{n}}$ converges to 0 . If the value of $\mathrm{D}_{\mathrm{n}}$ exceeds the critical value then $H_{0}$ is rejected. A table of thresholds for different values of $P_{f}$ is given in [20]. 


\section{c. Jarque and bera test}

The Jarque and Bera (JB) test is another goodness-of-fit test to check for normal distribution. It uses the skewness and kurtosis to determine whether the sample data is from a normal distribution [21]. The JB test statistic is the combination of the squares of normalized skewness and kurtosis and is given as follows:

$$
J=\frac{N}{6}\left(\gamma_{1}^{2}+\frac{\left(\gamma_{2}-3\right)^{2}}{4}\right)
$$

where, $\gamma_{1}$ is the skewness and $\gamma_{2}$ is the kurtosis and $N$ is the number of samples. The critical values of the JB test for different sample sizes are given in [21]. The primary user signal is declared present if the Jarque Bera test statistic is greater than the critical value and is declared as noise otherwise. The spectrum sensing problem using JB test can be expressed as

$$
\begin{aligned}
& H_{0}: J \leq \lambda_{c v} \\
& H_{l}: J>\lambda_{c v}
\end{aligned}
$$

\subsection{Significance of statistical measures}

\subsection{1. p-value}

Fisher justified that the $\mathrm{p}$-Value can be viewed as an index of the "strength of evidence" against $\mathrm{H}_{0}$, with small $p$ indicating an unlikely hypothesis [12]. The test statistic is used to determine the $p$-Value using the formula mentioned in Table 1 as given in [18] and the interpretation of the test results are given in Table 2 .

Table 1. The p-value formula for Anderson darling test

\begin{tabular}{cc}
\hline $\mathrm{AD}$ statistic & $\mathrm{p}$-Value Formula \\
\hline $\mathrm{A}>153.467$ & $p=0$ \\
$0.6<\mathrm{A} \leq 153.467$ & $p=e^{\left(1.2937-5.709 * A+0.0186 A^{2}\right)}$ \\
$0.34<\mathrm{A} \leq 0.60$ & $p=e^{\left(0.9177-4.279 * A-1.38 A^{2}\right)}$ \\
$0.20<\mathrm{A} \leq 0.34$ & $p=1-e^{\left(-8.318+42.796 * A-59.938 A^{2}\right)}$ \\
$\mathrm{A} \leq 0.20$ & $p=1-e^{\left(-13.436+101.14 * A-223.73 A^{2}\right)}$ \\
\hline
\end{tabular}

Table 2. Decision table

\begin{tabular}{ccc}
\hline Method & Condition & Decision \\
\hline Classical test & if( test statistic $>$ Critical value $)$ & $\mathrm{H}_{0}$ is rejected \\
Classical test & if (test statistic $<$ Critical value $)$ & $\mathrm{H}_{0}$ cannot be rejected \\
$\mathrm{p}-$ Value & $(\mathrm{p}-$ Value $<\alpha)$ & $\mathrm{H}_{0}$ is rejected \\
$\mathrm{p}-$ Value & $(\mathrm{p}-$ Value $>\alpha)$ & $\mathrm{H}_{0}$ cannot be rejected \\
\hline
\end{tabular}

The steps involved in hypothesis testing using p-Values given in [12] are as follows:

a. Define the null and alternative hypotheses.

b. Compute the test statistic from the sample data.

c. Determine the $\mathrm{p}-$ Value using the test statistic obtained from step 2.

d. Fix the significance level $\alpha=0.05$ and interpret the results using Table 2 .

\subsubsection{Bayes factor method}

The term Bayes factor (BF) is also called as likelihood ratio. The Bayes factor is often referred to as the "strength of evidence" or "weight of evidence". Bayes factors show that p-Values greatly overstate the evidence against the null hypothesis. The Bayes factors have direct interpretation as the strength of the evidence in favor of $\mathrm{H}_{1}$ relative to $\mathrm{H}_{0}$. The use of Bayes factors can avoid the misinterpretations that arise from dependency on the $\mathrm{p}-$ Value in decisions [13, 14]. Minimum Bayes factors have the advantage that they do not depend on the prior probability. The proof of the minimum Bayes factor as function of the $\mathrm{p}$-Value is given in [14]. Figure 3 shown Categorization of Bayes Factors $\mathrm{BF}<1$ into levels of evidence against $\mathrm{H}_{0}$.

$$
\min B F(p)=\left\{\begin{array}{c}
- \text { eplogp for } p<\frac{1}{e} \\
1 \text { otherwise }
\end{array}\right\}
$$


Table 3. Categorization of bayes factors $\mathrm{BF}<1$ into levels of evidence against $\mathrm{H}_{0}$ as given in [14]

\begin{tabular}{cc}
\hline Strength of Evidence & Bayes factor \\
\hline Weak & 1 to $1 / 3$ \\
Moderate & $1 / 3$ to $1 / 10$ \\
Substantial & $1 / 10$ to $1 / 30$ \\
Strong & $1 / 30$ to $1 / 100$ \\
Very strong & $1 / 100$ to $1 / 300$ \\
Decisive & $<1 / 300$ \\
\hline
\end{tabular}

\section{PROPOSED METHOD}

The block diagram of the proposed method is given in Figure 1. This paper adopts the following statistical methods to integrate the statistical measures from independent tests [22-25] to have an overall assessment on the detection of the primary user signal activity.

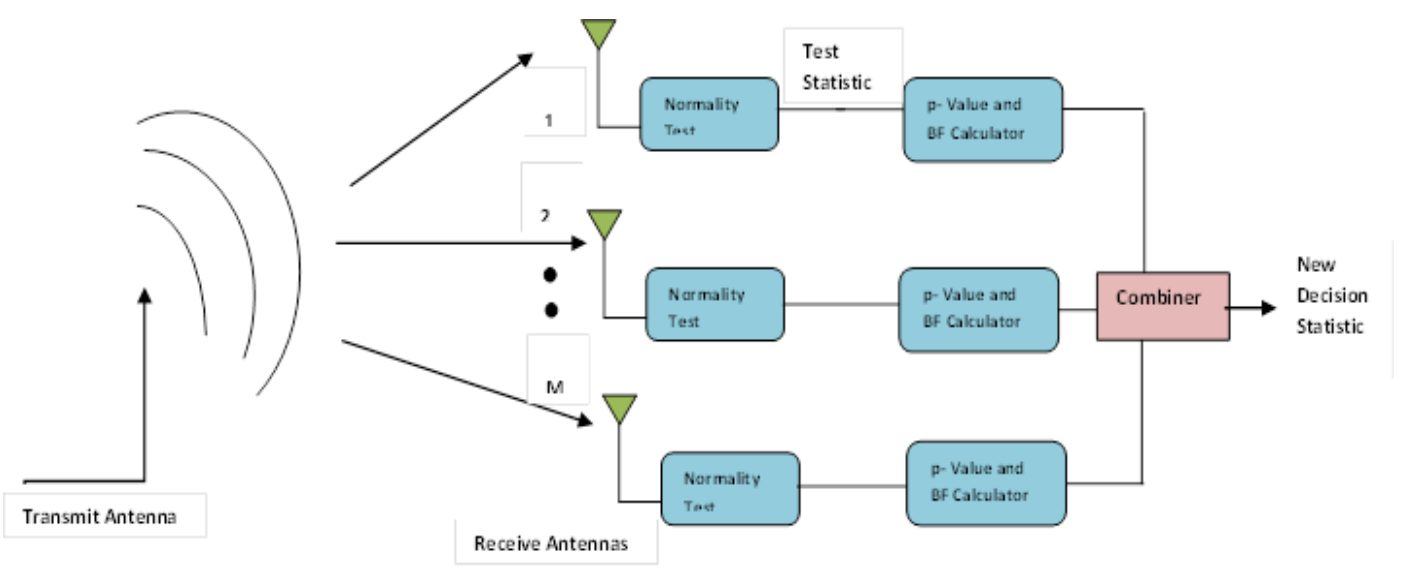

Figure 1. Block diagram of the proposed method

\section{1. p-value based diversity combiner}

The $M$ independent samples $x_{i}[k] i=1,2 \ldots M$ are received from $M$ diversity branches of the CR receiver. The test statistics $\left(\mathrm{A}_{1}, \mathrm{~A}_{2} \ldots . \mathrm{A}_{\mathrm{M}}\right)$ and its corresponding $\mathrm{p}$-Values $\left(\mathrm{p}_{1}, \mathrm{p}_{2} \ldots . . \mathrm{p}_{\mathrm{M}}\right)$ are computed. This study adopts the following statistical methods to integrate the $\mathrm{p}$-Values from independent tests [22, 23] to have an overall assessment on the detection of the primary user signal activity.

\subsubsection{Fisher's test}

A popular method of combining the p-Values is the Fisher's method [22]. Let $\mathrm{p}_{1}, \mathrm{p}_{2}, \ldots, \mathrm{p}_{\mathrm{M}}$ be the significance probabilities of the test statistic A or $\mathrm{J}$ from the $\mathrm{i}^{\text {th }}$ sample received from each diversity branch of the CR receiver. The joint assessment of the normality is based on the $M$ values of the statistic. The different significance probabilities obtained from $M$ diversity branches are combined using Fisher's method as given below.

$$
F_{T}=-2 \ln \left(\sum_{i=1}^{M} p_{i}\right)
$$

\subsection{Bayes Factor based diversity combiner}

From the values $\left(\mathrm{p}_{1}, \mathrm{p}_{2} \ldots . . \mathrm{p}_{\mathrm{M}}\right)$ their corresponding Minimum Bayes factors are computed from (13). In the context of spectrum sensing the following method is proposed to integrate these statistical measures from independent tests [24] to have an overall assessment on the detection of the primary user signal activity. The method proposed for combining the data is by calculating the product of the Bayes factor calculated from $M$ independent samples and is defined as the Group Bayes Factor (GBF) as given in [24].

$$
G B F_{i j}=\prod_{n=1}^{M} B F_{i, j}^{(n)}
$$

where, the subscripts $i, j$ refer to the hypothesis models being compared, and the bracketed superscript refers to the $M$-th sample. Since the measured data is treated as conditionally independent samples, the probabilities are multiplied. 


\section{Algorithm 1. Statistical approach to spectrum sensing}

1. Obtain $M$ observation samples from each of the diversity branches of the CR node.

2. Let $Z_{i},(i=1 \ldots M)$ be the observation vector. Sort the observations from each branch in ascending order.

3. Calculate the AD test statistic using Equation (7) and (8). Let $A_{i}(i=1 \ldots M)$ denote the test statistic obtained for $M$ diversity branches.

4. Using the formula given in Table 1 calculate the $\mathrm{p}$-Value $\mathrm{p}_{1} \mathrm{p}_{2}, \ldots, \mathrm{p}_{\mathrm{M}}$ and their respective MBFs using Equation (13).

5. The MBFs and $\mathrm{p}$-Values from $M$ diversity branches are combined using Equation (14) and (15) to obtain the new decision statistic.

6 Reject null Hypothesis if the new decision statistic is less than the predefined significance level.

\section{RESULTS AND ANALYSIS}

\subsection{Monte carlo simulations}

The performance analysis of spectrum sensing using receiver diversity in a CR environment are carried out using 1) Conventional Hypothesis Testing and 2) Statistical Hypothesis testing. The statistical hypothesis testing is carried out using the following two methods 1) p-Values 2) Minimum Bayes Factor.

The detection probability is used as a standard of measurement to determine the sensing accuracy. The following assumptions are made in the simulations.

a. The system model has Single Input Multiple Output.

b. The primary transmitter signal is a sinusoidal pilot signal of known frequency.

c. Additive White Gaussian Noise with $\mu=0$ and $\sigma^{2}=1$.

d. For the Hypothesis $\mathrm{H}_{1}$ to be declared true (signal is present)

Method 1: If the $\mathrm{p}$-Values is less than the significance level $\alpha=0.05$

Method 2: If the minimum Bayes factor is less than 1/100

Figure 2 provides a comparison of the Goodness of fit tests in the context of primary user signal detection in cognitive radio. The Conventional method of hypothesis testing in a CR environment is compared with the statistical method of hypothesis testing. The number of samples in the test is taken as 100 . The tests include the the conventional energy detector, Anderson Darling test, Kolmogorov-Smirnov Test and Jarque and Bera Test. Energy detector shows better performance compared to the other tests. But AD test provides better detection performance compared to the other normality tests. The statistical measure coined as p-Value and Bayes Factor are used as statistical measures in hypothesis testing in Figures 3-6.

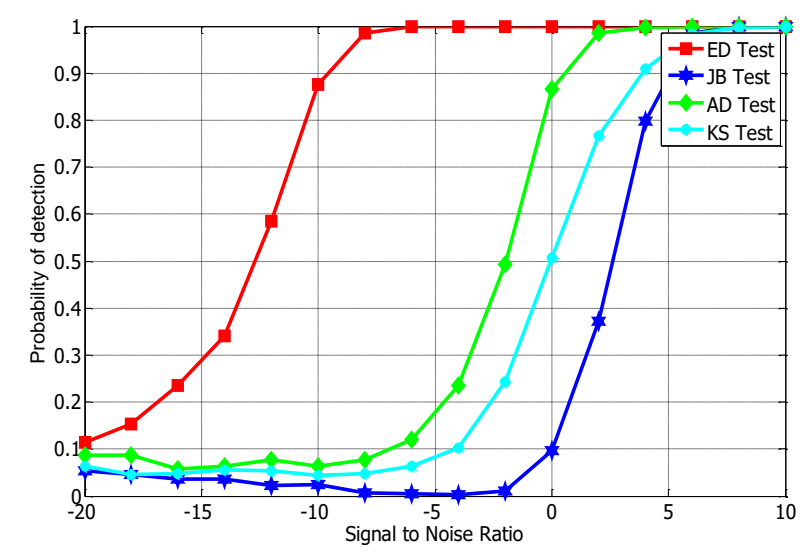

Figure 2. Spectrum sensing using Goodness of fit tests when the primary user is present

The following observations are made:

a. It is observed that the p-Value method overstates the evidence against the null hypothesis than the MBF method.

b. The diversity improves the detection probability in the low SNR regimes using both the methods.

c. The statistical methods provide detection probability close to the conventional square law methods of combining. 


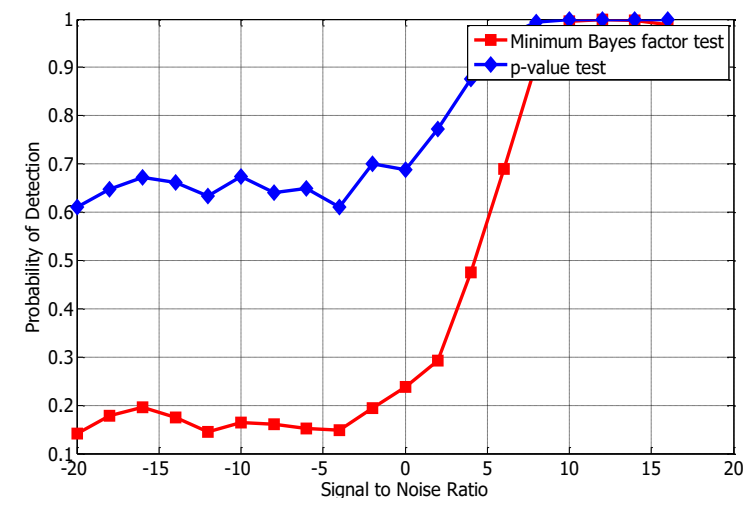

Figure 3. Primary User detection of a Sinusoidal Pilot signal with $\mathrm{M}=1, \alpha=0.05$ and $\mathrm{N}=100$ Samples using Anderson Darling Statistic

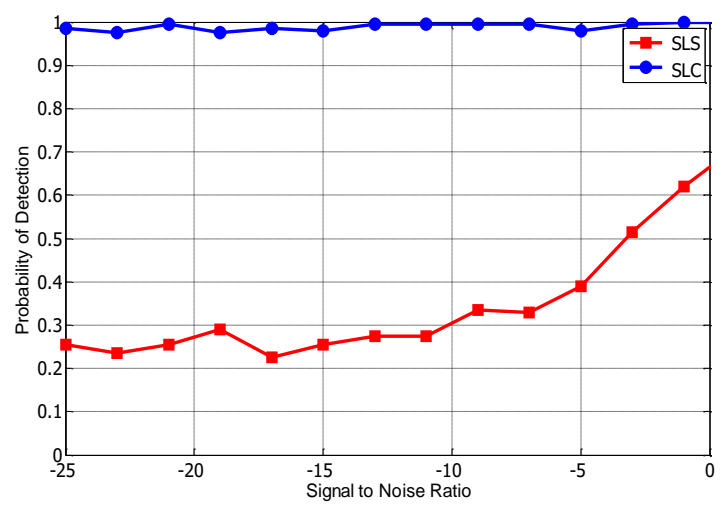

Figure 5. Primary User detection of a Sinusoidal Pilot signal with $\mathrm{M}=3, \mathrm{P}_{\mathrm{f}}=0.05$ and $\mathrm{N}=100$ Samples using

Conventional Square Law diversity combining techniques

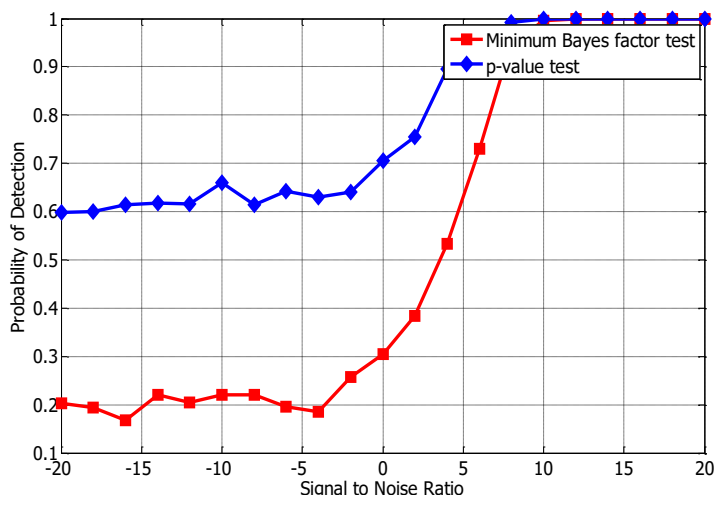

Figure 4. Primary User detection of a Sinusoidal Pilot signal with $\mathrm{M}=1, \alpha=0.05$ and $\mathrm{N}=100$ Samples using Jarque Bera Statistic

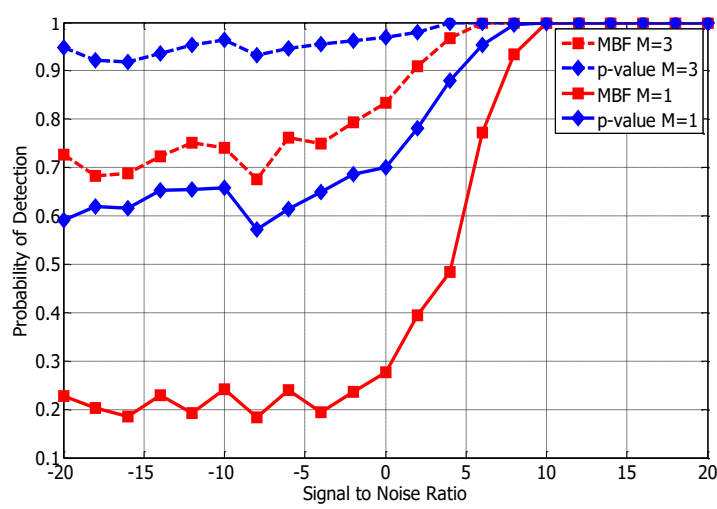

Figure 6. Primary User detection of a Sinusoidal Pilot signal with $\mathrm{M}=3, \alpha=0.05$ and $\mathrm{N}=100$ Samples using statistical approach a)Minimum Bayes Factor method b) p- Value Method

Given below is a detailed illustration to support point 1 of the observation:

If the $\mathrm{MBF}=0.262788=1 / 3.8$ with $\mathrm{p}$-value $=0.070552$ then based on the observed evidence, the result is that the $\mathrm{H}_{1}$ (the alternative hypothesis) is 3.8 times as likely as $\mathrm{H}_{0}$ (the null hypothesis). Since MBF/p-Value $=0.262788 / 0.070552$ it can be seen that Fisher's $p$-Value states the amount of evidence against $\mathrm{H}_{0}$ as 3.7 times as much as the MBF does. This means that the exaggeration of the statistical significance by Fisher' $p$-Value is almost 4 times as much as that of MBF. Therefore it can be stated that Fisher's p-Value provides less accurate results as a measure of the strength of evidence against $\mathrm{H}_{0}$ [14].

\section{CONCLUSION}

In this paper a novel Statistical approach for the hypothesis testing problem in a spectrum sensing environment is proposed. The Minimum Bayes factors and $p$-Values are proposed for combining the data received from a secondary user equipped with multiple antennas. The effect of these statistical measures in sensing the spectrum in a Cognitive Radio environment is investigated. A ballpark figure of the merits of these diversity combining methods are provided in this study. Results show that p-Values magnifies the evidence against the null hypothesis. The Bayes factor has a straightforward interpretation as the strength of the evidence in favor of $\mathrm{H}_{1}$ relative to $\mathrm{H}_{0}$. Also the algorithm proposed improves the detection of the PU in low SNR regimes. Through extensive Monte Carlo simulations it is shown that Bayes Factors provides a promising solution to combine the test statistics from multiple receiver antennas and can be used as an alternative to the conventional hypothesis testing methods for spectrum sensing. Hence this novel statistical approach using bayes factors provide more accurate and relevant test results when measuring the strength of the evidence against the hypothesis. 


\section{REFERENCES}

[1] S. Atapattu, et al., "Energy detection based cooperative spectrum sensing in cognitive radio networks," IEEE Transactions on Wireless Communications, vol. 10, pp. 1232-1241, 2011.

[2] Yucek T., et al., "A survey of spectrum sensing algorithms for cognitive radio applications," IEEE communications surveys \& tutorials, vol. 11, pp. 116-30, 2009.

[3] Mietzner J., et al., "Multiple-antenna techniques for wireless communications-a comprehensive literature survey," IEEE communications surveys \& tutorials, vol. 11, pp. 87-105, 2009.

[4] Wang P., et al., "Multiantenna-assisted spectrum sensing for cognitive radio," IEEE Transactions on Vehicular Technology, vol/issue: 59(4), pp. 1791-800, 2010.

[5] Digham F. F., et al., "On the energy detection of unknown signals over fading channels," IEEE transactions on communications, vol. 55, pp. 21-24, 2007.

[6] Annavajjala R., et al., "Performance analysis of linear diversity-combining schemes on Rayleigh fading channels with binary signaling and Gaussian weighting errors," IEEE Transactions on Wireless Communications, vol. 4, pp. 2267-78, 2005.

[7] Herath S. P., et al., "On the energy detection of unknown deterministic signal over Nakagami channels with selection combining," IEEE Canadian conference on the Electrical and Computer Engineering, pp. 745-749, 2009.

[8] Akbari M., et al., "Receiver diversity combining using evolutionary algorithms in rayleigh fading channel," The Scientific World Journal, 2014.

[9] Arshad K., et al., "Robust spectrum sensing based on statistical tests," IET Communications, vol. 7, pp. 808-17, 2013.

[10] Carvalho F. B., et al., "Cognitive spectrum sensing based on statistical tests in fading channels," Communications (LATINCOM), 7th IEEE Latin-American Conference, pp. 1-6, 2015.

[11] H. Wang, et al., "Spectrum sensing in cognitive radio using goodness of fit testing," IEEE Transactions on Wireless Communications, vol/issue: 8(11), 2009.

[12] Berger J. O., "Could Fisher, Jeffreys and Neyman have agreed on testing?" Statistical Science, vol. 18, pp. 1-32, 2003.

[13] Katki H. A., "Invited commentary: evidence-based evaluation of p values and Bayes factors," American Journal of Epidemiology, vol. 168, pp. 384-388, 2008.

[14] Held L., et al., "How the maximal evidence of p-values against point null hypotheses depends on sample size," The American Statistician, vol. 70, pp. 335-41, 2016.

[15] Goodman S. N., "Toward evidence-based medical statistics. 1: The P value fallacy," Annals of internal medicine, vol. 130, pp. 995-1004, 1999.

[16] S. N. Goodman, "Toward evidence-based medical statistics. 2: The Bayes factor," Annals of internal medicine, vol. 130, pp. 1005-1013, 1999.

[17] Romeu J. L., "Anderson-Darling: a goodness of fit test for small samples assumptions,” RAC START, 2003.

[18] R. B. D'Agostino and Stephens M. A., "Goodness-of-fit techniques," Statistics: Text books and monograph, Boca Raton, Florida: CRC Press, 1986.

[19] Stephens M. A., "EDF statistics for goodness of fit and some comparisons," Journal of the American statistical Association, vol. 69, pp. 730-7, 1974.

[20] S. Facchinetti, "A procedure to find exact critical values of Kolmogorov-Smirnov test," Statistica Applicata, vol. 21, pp. 337-359, 2009.

[21] T. Thadewald and H. Büning, "Jarque-Bera test and its competitors for testing normality-a power comparison," Journal of Applied Statistics, vol. 34, pp. 87-105, 2007.

[22] Bhandary M., et al., "Comparison of several tests for combining several independent tests," Journal of Modern Applied Statistical Methods, vol. 10, 2011.

[23] Whitlock M. C., "Combining probability from independent tests: the weighted Z-method is superior to Fisher's approach," Journal of evolutionary biology, vol. 18, pp. 1368-73, 2005.

[24] Stephan K. E., et al., "Bayesian model selection for group studies. Neuroimage," vol. 46, pp. 1004-17, 2009.

[25] Pettitt A. N., "Testing the normality of several independent samples using the Anderson-Darling statistic," Applied Statistics, pp. 156-61, 1977. 